\title{
Brain lesions in the course of generalised tetanus
}

\author{
T lijichi, T Yamada, S Yoneda, Y Kajita, K Nakajima, M Nakagawa
}

J Neurol Neurosurg Psychiatry 2003;74:1432-1434

\begin{abstract}
A 47 year old woman developed left hemiparesis primarily affecting the lower limbs during the course of severe generalised tetanus. MRI on the 82 nd hospital day revealed cortical and subcortical lesions predominantly in the right frontal and parietal lobes in addition to marked brain atrophy. Three months later, the enhancing lesions were still present on follow up MRI. We postulate that structures above brainstem may be involved in severe generalised tetanus.
\end{abstract}

$\mathrm{T}$ etanus is an exotoxin mediated disease caused by Clostridium tetani, an anaerobic, Gram positive, spore forming rod. Tetanus toxin produced by this organism exerts its effect on the spinal cord, brainstem, and at neuromuscular junctions. ${ }^{12}$ The toxin reaches the CNS by retrograde axonal transport through motor and vegetative efferent axons. ${ }^{3}$ Once transported into the CNS, the toxin can continue rostal movement by retrograde transport. ${ }^{4}$ This implies the possibility of involvement of structures above the brainstem by tetanus toxin. ${ }^{1}$ We present a patient with generalised tetanus who developed left hemiparesis with unusual brain imaging findings. Our observations suggest the involvement of structures above brainstem in generalised tetanus.

\section{CASE REPORT}

A healthy 47 year old woman sustained a deep laceration $4 \mathrm{~cm}$ in length below the left knee when she accidentally fell from a bicycle. Soon afterward the wound was treated at a hospital. Three days later she developed neck and back pain, and noted difficulty in opening her mouth. With these symptoms worsening, she visited our hospital three days after the onset of the symptoms. On her arrival, vital signs were: blood pressure, 110/70 $\mathrm{mm} \mathrm{Hg}$; pulse, 72/min; respiratory rate, 18 breaths $/ \mathrm{min}$; and body temperature, $36.6^{\circ} \mathrm{C}$. The patient's chest and abdomen were normal. "Risus sardonicus" was present and she could not open her mouth. The shoulders and back showed muscular rigidity. Muscle strength and deep tendon reflexes were normal. Routine blood tests were normal. Arterial blood gas analysis demonstrated pH 7.422, $\mathrm{PCO}_{2} 38.6$ Torr, and $\mathrm{PO}_{2}$ 64.1 Torr. The wound showed erythema and drainage, and was debrided by a surgeon. The patient was uncertain if she had ever been immunised for tetanus.

The patient was diagnosed with tetanus and admitted to the intensive care unit. Within $6 \mathrm{~h}$, she developed muscular spasm, dysphagia, and dyspnea. She required ventilatory support via a tracheostomy, sedation with benzodiazepines, and neuromuscular blockades. Sinus tachycardia and hypertension also developed, reflecting autonomic involvement. Disease manifestations were so severe and prolonged that ventilatory support and sedation were needed for 45 days. No major complications occurred from mechanical ventilation. When a rehabilitation programme was begun, she complained of weakness involving the left limbs. She was referred to a neurologist. Neurologic examination demonstrated a left hemiparesis predominantly affecting the lower limb and mild impairment of cognitive functions such as attention and memory. A Babinski sign was present. Motor and sensory peripheral nerve conduction studies were normal. A brain CT showed marked brain atrophy for her age and low density areas in the right frontal and parietal lobes. On the 82nd hospital day, non-contrast brain MRI showed areas of low intensity on Tl weighted images and high intensity on T2 weighted images mainly in the right frontal and parietal lobes (fig 1). There were no abnormal signals in watershed territories. Brain magnetic resonance angiography showed no abnormalities. MRI with gadolinium on the 95th hospital day demonstrated enhancement of the lesions on the right frontal and parietal cortex and subcortical regions (fig $2 \mathrm{~A}, \mathrm{~B}$, and $\mathrm{C}$ ). MRI of the spinal cord and brainstem were unremarkable. Further history taking was negative for drugs of misuse and oral contraceptives. No family history of stroke was noted. Serum levels of protein C, protein S, and antithrombin III were normal, and no IgM or IgG antibodies against cardiolipin were detected. Autoantibodies such as antinuclear antibody or anti-DNA antibody were absent. Transthoracic echocadiogram with a microbubble study showed no abnormalities suggesting a patent foramen ovale, which is sometimes associated with brain embolism from deep venous thrombosis. Three months later, the enhancing lesions were still present on follow up MRI (fig 2D, E, and F). Recovery from the left hemiparesis and the cognitive impairment was partial.

\section{DISCUSSION}

The effects of tetanus toxin on the spinal cord and brainstem are due to the inhibition of $\gamma$-aminobutyric acid (GABA) and glycine transmission between inhibitory neurons and motoneurons. ${ }^{5}$ Cortical GABAergic inhibitory networks probably exist in humans, ${ }^{6}$ however, to what extent structures above brainstem are involved in patients with tetanus remains unknown. $^{2}$ To our knowledge, there have been few clinical reports describing the possibility of this involvement. Warren et al reported the involvement of the cortex in a patient with generalised tetanus by physiologic study. Transcranial magnetic brain stimulation in their patient revealed enlarged electromyographic (EMG) responses and absence or reduction of the late phase of EMG silence following the motor evoked potential. They proposed that the disruption of central nervous inhibitory mechanisms in generalised tetanus might be widespread. Barlow et $a l^{8}$ reported that children who survived neonatal tetanus had evidence of brain damage that manifested as microcephaly, mild neurologic abnormalities, and developmental impairment.

The manifestations of our patient such as muscular spasm and autonomic involvement are consistent with generalised

Abbreviations: GABA, $\gamma$-aminobutyric acid; EMG, electromyographic 


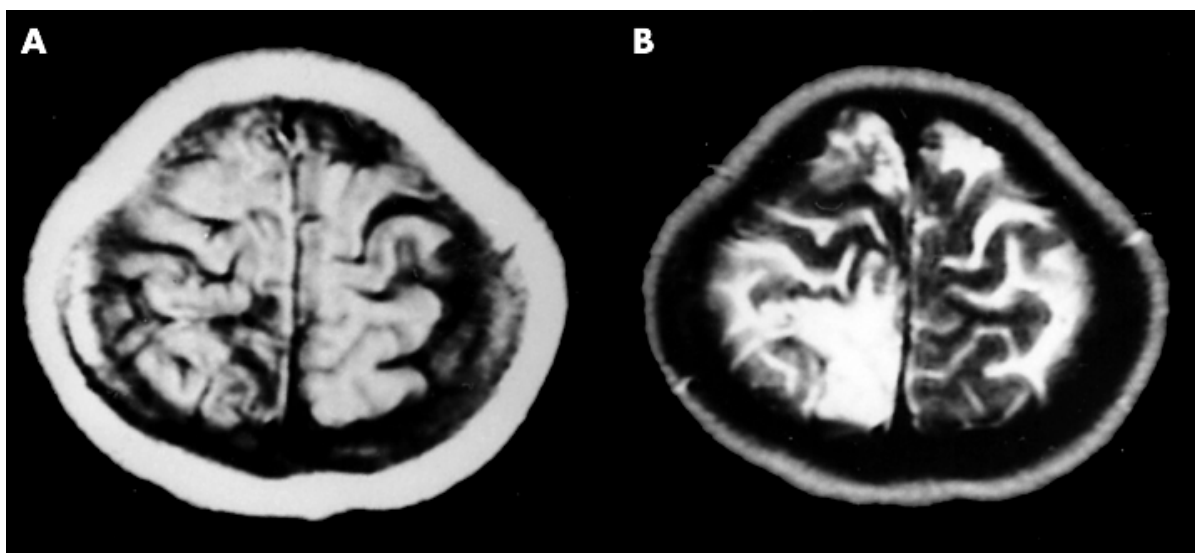

Figure 1 Axial brain MRI on the 82nd hospital day showing low intensity areas on $\mathrm{T} 1$ weighted images (A), which take on high intensity on T2weighted images (B) in the right frontal and parietal lobes including areas near the central sulcus. Brain atrophy is also noted.

tetanus. ${ }^{12}$ In contrast, hemiparesis in the course of tetanus is uncommon. The brain lesions in our patient were not likely to represent ischemic infarction for at least three reasons. Firstly, the patient had no risk factors for brain infarction before the admission. She had no history of hypertension, diabetes mellitus, drug misuse, or oral contraception. Symptoms, signs, and laboratory data supporting diagnosis of collagen disease or coagulation abnormality were absent. Brain infarction in such a middle aged woman is uncommon. Secondly, we started ventilatory support before the onset of any severe hypoxia. No complications except for sinus tachycardia occurred that could have caused brain ischaemia such as hypotension, hypoxia, or severe ventricular arrhythmia. Thirdly, enhancement of lesions on MRI was still present three months later. This finding is not consistent with brain infarction because the enhancement may be seen in a recent but not an old infarct. ${ }^{9}$ Thus, we speculate that the lesions were associated with causes other than brain infarction. The enhancing lesions were not periventricular but were associated with cortical lesions. This finding is uncommon in demyelinating diseases such as multiple sclerosis. We also speculate that brain atrophy in our patient occurred in the course of tetanus because she developed cognitive impairment after admission. Furthermore, it is interesting that the enhancing lesions on MRI in our patient were present in the right frontal lobes near the central sulcus, an area that includes upper motor neurons monosynaptically or multisynaptically connected to anterior horn cells innervating muscles below the left knee, where the patient sustained the infected wound. From this topography and brain atrophy, the brain lesions may be associated with the disruption of central nervous inhibitory mechanisms by
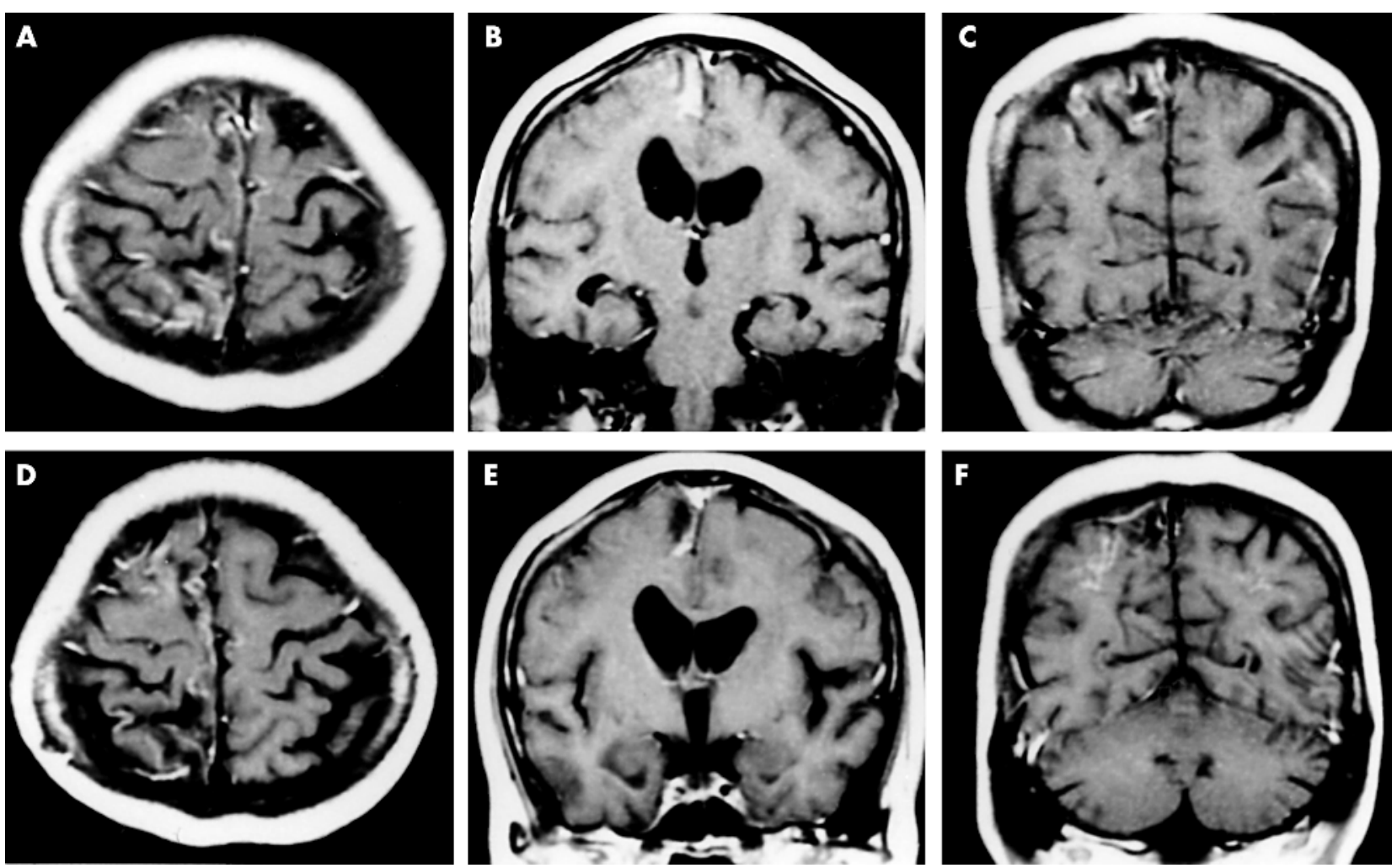

Figure 2 (A) Gadolinium enhanced brain MRI on the 95th hospital day showing enhancing lesions on an axial view. (B, C) Enhancing lesions are noted in the parasagittal area on coronal views. (D, E, and F) On follow up MRI on the 185th hospital day, the enhancing lesions are still present. (B, E) The centrum semiovale of the right side appears more atrophic than on the left. 
tetanus toxin as proposed by Warren et al. ${ }^{7}$ The amount of toxin in our case may have been large enough to gain access to inhibitory pathways above brainstem considering the severity of the clinical course. Our observations suggest that tetanus may have a broader clinical spectrum of CNS involvement than previously realised.

\section{Authors' affiliations}

T ljichi, Department of Neurology Nantan General Hospital, Kyoto,

Japan

T Yamada, S Yoneda, Y Kajita, Department of Internal Medicine,

Nantan General Hospital, Japan

K Nakajima, M Nakagawa, Department of Neurology, Kyoto

Prefectural University of Medicine,Kyoto, Japan

Correspondence to: Dr T liichi, Department of Neurology, Kyoto Prefectural University of Medicine, 465 Kajii-cho, Kawaramachi Hirokoji, Kamigyo-ku, Kyoto 602-8566, Japan; tijichi@koto.kpu-m.ac.jp

Competing interests: none declared

\section{REFERENCES}

1 Bleck TP. Tetanus. In: Scheld WM, Whitley RJ, Durack DT, eds. Infection of the central nervous system. New York: Raven Press, 1991:603-24.

2 Farrar JJ, Yen LM, Cook T, et al. Neurological aspects of tropical disease: tetanus. J Neurol Neurosurg Psychiatry 2000;69:292-301.

3 Girod J, Fenart L, Régina A, et al. Transport of cationized anti-tetanus Fab'2 fragment across an in vitro blood-brain barrier model. Involvement of the transcytosis pathway. J Neurochem 1999:73:2002-8.

4 Dumas $M$, Schwab ME, Thoenen $H$. Retrograde axonal transport of specific macromolecules as a tool for characterizing nerve terminal membranes. $J$ Neurobiol 1979;10:179-97.

5 Menestrina G, Schiavo G, Montecucco C. Molecular mechanisms of action of bacterial protein toxins. Mol Aspects Med 1989;15:181-93.

6 Ziemann U, Lonnecker S, Steinhoff BJ, et al. Effects of antiepileptic drugs on motor cortex excitability in humans: a transcranial magnetic stimulation study. Ann Neurol 1996:40:367-78.

7 Warren JD, Kimber TS, Thompson PD. The silent period after magnetic brain stimulation in generalized tetanus. Muscle Nerve 1999;22:1590-2.

8 Barlow JL, Mung'Ala-Odera V, Gona J, et al. Brain damage after neonatal tetanus in a rural Kenyan hospital. Trop Med Int Health $2001 ; 6: 305-8$

9 Miyashita K, Naritomi H, Sawada T, et al. Identification of recent lacunar lesions in cases of multiple small infarction by magnetic resonance imaging. Stroke 1988;19:834-9. 